\title{
Estimulación de corriente continua transcraneal e intervención logopédica en personas con afasia: revisión sistemática de la bibliografía
}

\author{
Carmen Corrales-Quispiricra, M. Engracia Gadea, Raúl Espert
}

Introducción. La recuperación de la afasia podría requerir terapias adyuvantes a la rehabilitación logopédica. La estimulación de corriente continua transcraneal (tDCS) es una técnica neuromoduladora no invasiva que se puede usar para mejorar la actividad cerebral cortical.

Objetivos. Ofrecer una visión general sobre la tDCS en personas con afasia desde una visión logopédica.

Pacientes y métodos. Se han analizado 35 estudios que realizaban intervenciones online (la aplicación de la tDCS junto con cualquier tipo de terapia de la afasia), así como intervenciones offline (realización de entrenamiento logopédico antes o después de la tDCS). Los estudios se publicaron durante los últimos 10 años (enero de 2009 a enero de 2019). En el análisis se revisaron las bases de datos PubMed, Scopus y Embase.

Resultados. La mayoría de los estudios incluye muestras heterogéneas, sin tener en cuenta la sintomatología propia de cada tipo de afasia y sin individualizar los tratamientos empleados. La anomia es el déficit lingüístico que más se ha tratado.

Conclusiones. Es mejor realizar la tDCS junto con terapia logopédica online. La tDCS es una técnica que puede ayudar a mejorar los déficits del lenguaje de las personas con afasia. Se deben realizar intervenciones logopédicas basadas en conocimientos neurocientíficos que tengan una repercusión en la vida cotidiana de la personas con afasia.

Palabras clave. Afasia. Estimulación eléctrica transcraneal por corriente continua. Ictus. Lenguaje. Logopedia. tDCS.

\section{Introducción}

La afasia se define como 'un trastorno del lenguaje y de la comunicación que surge como consecuencia de una lesión que afecta a las redes del lenguaje en el cerebro, que son responsables de los procesos de producción y comprensión en sus diversos niveles y componentes' [1]. La mayoría de las personas afectadas experimenta una recuperación espontánea durante los tres primeros meses posteriores al ictus [2]. Esta recuperación espontánea tiende a estabilizarse durante el primer año después del ictus, con la consiguiente persistencia de las alteraciones del lenguaje en las etapas crónicas [3]. Por eso los déficits crónicos del lenguaje requieren rehabilitación [4].

La recuperación de la afasia necesita una reorganización de las estructuras corticales que sustentan el habla y el lenguaje [5], las cuales están conectadas a través de la ruta dorsal implicada en funciones fonológicas (repetición de pseudopalabras) y la ruta ventral encargada de los aspectos semánticos [6,7]. El proceso dinámico de recuperación del lenguaje puede involucrar una variedad de cambios plásticos en ambos hemisferios [8]. En este sentido, se han desarrollado tres modelos básicos de neuroplasticidad en relación con la recuperación de la afasia: re- clutamiento beneficioso de áreas perilesionales que rodean los tejidos infartados en el hemisferio dominante, reclutamiento beneficioso o desinhibición de áreas del lenguaje homotópicas en el hemisferio no dominante y activación de centros de lenguaje ineficientes o perjudiciales en el hemisferio derecho que pueden interferir con la recuperación de redes del lenguaje en el hemisferio izquierdo [8-10]. Por otro lado, según la teoría de desinhibición transcallosa, los dos hemisferios del cerebro normalmente están regulados y equilibrados de manera inhibitoria. Sin embargo, este equilibrio se ve interrumpido cuando se produce un daño cerebral. Así pues, si el daño se produce en el hemisferio izquierdo, como consecuencia se produce un aumento de la inhibición del hemisferio derecho sobre el izquierdo. Esto es así, ya que una lesión unilateral del lado izquierdo reduce la inhibición transcallosa del hemisferio derecho por el hemisferio izquierdo, y aumenta entonces la actividad en el hemisferio derecho intacto. Dado que el hemisferio derecho todavía puede enviar impulsos inhibidores transcallosos al hemisferio izquierdo, la activación de éste se reduce aún más [11] y puede provocar una mala recuperación del lenguaje [12]. Además de las diferentes teorías sobre los mecanismos de neuroplasticidad, algunas inves-
Unidad de Neuropsicología; Hospital Clínico Universitario de Valencia (C. Corrales-Quispiricra, M.E. Gadea, R. Espert). Departamento de Psicobiología; Facultad de Psicología; Universitat de València (M.E. Gadea, R. Espert). Valencia, España.

Correspondencia:

Dr. Raúl Espert Tortajada. Departamento de Psicobiología. Facultad de Psicología. Universitat de València. Avda. Blasco Ibáñez, 21. E-46010 Valencia.

E-mail:

raul.espert@uv.es

Aceptado tras revisión externa: 07.02.20.

Cómo citar este artículo: Corrales-Quispiricra C, Gadea ME, Espert R. Estimulación de corriente continua transcraneal e intervención logopédica en personas con afasia: revisión sistemática de la bibliografía. Rev Neurol 2020; 70: 351-64. doi: 10.33588/rn.7010.2019397.

(c) 2020 Revista de Neurología 
tigaciones sugieren que la recuperación del lenguaje depende de varios factores clínicos, como la extensión y la ubicación de la lesión [9]. Otros autores sugieren que la participación del hemisferio derecho en la fase crónica, después del ictus, depende de la cantidad de lateralización del lenguaje premórbido [13]. Por ello, las personas con una representación del lenguaje premórbido más bilateral utilizan más áreas homologas.

La capacidad del cerebro humano para cambiar de manera flexible la funcionalidad dentro de una red es una característica fundamental de la reorganización adaptativa y la compensación después de las lesiones cerebrales [14]. Esta reorganización cerebral podría requerir terapias adyuvantes a la rehabilitación logopédica, sobre todo en etapas crónicas del ictus [15].

Las técnicas de estimulación cerebral no invasiva, que incluyen tanto la estimulación magnética transcraneal, la estimulación magnética repetitiva y la estimulación transcraneal de corriente continua (tDCS), son enfoques que podrían ayudar en la recuperación de la afasia [15].

Concretamente, hay evidencia que sugiere que la tDCS puede mejorar los efectos de la terapia del lenguaje en personas con afasia al facilitar la reestructuración neuronal después de la pérdida de la función tras una lesión cerebral $[16,17]$.

La tDCS es una técnica neuromoduladora no invasiva que se puede usar para modificar la actividad cerebral cortical, así como para inducir cambios de comportamiento medibles [18]. La administración de la tDCS implica la aplicación de corrientes eléctricas de baja intensidad, a través de electrodos de superficie colocados sobre el cuero cabelludo. Hay un electrodo que se utiliza para estimular; dicho electrodo se conoce como electrodo activo, polo positivo o estimulación anódica (A-tDCS), ya que provoca potenciales postsinápticos excitatorios; hay otro electrodo, denominado polo negativo o estimulación catódica (C-tDCS), cuya acción es la de inducir potenciales postsinápticos inhibitorios. Recientemente se ha introducido la tDCS de alta definición o electrodos de anillo, estos comprenden cinco electrodos pequeños, como un único ánodo rodeado de cuatro cátodos, o viceversa [19]. Se ha demostrado que este tipo de montaje es factible y seguro, y puede inducir cambios duraderos en la excitabilidad del cerebro [20].

Asimismo, hay distintos montajes, pero los dos más utilizados son el bihemisférico, que utiliza la colocación de un electrodo anódico en el área perilesional y un electrodo catódico en el área contralesional, y el monocefálico, el cual se emplaza en el área perilesional y un electrodo de referencia (normalmente en una zona extracefálica como la cara o el hombro) [11]. Por otro lado, la tDCS a menudo se conceptualiza como una técnica neuromoduladora en lugar de neuroestimuladora [15], ya que ella no genera potenciales de acción, sino que interactúa con la actividad neuronal online en el cerebro [21]. Por ello, según Alharbi et al [18], la tDCS debería combinarse con terapia logopédica para obtener un efecto terapéutico en una habilidad específica. La tDCS es segura, pues no tiene efectos adversos relevantes, siempre que los parámetros de estimulación se mantengan dentro de los límites de seguridad [22,23]. Además, se ha demostrado que la tDCS puede producir mejoras significativas en diferentes patologías, por ejemplo, en el estado de ánimo [24], en la enfermedad de Parkinson [25] y en el dolor crónico [26]. En personas sanas, algunos estudios han demostrado que la A-tDCS mejora la fluidez verbal [27] y la velocidad verbal $[28,29]$.

Ante el panorama de nuevos métodos de intervención en personas con afasia, el objetivo de esta revisión es ofrecer una visión general sobre la tDCS en esta población. Para ello se han planteado las siguientes preguntas de investigación: ¿qué tipo de afasia se interviene?, ¿qué déficits afásicos se abordan?, ¿qué tipo de terapia logopédica se debe aplicar conjuntamente con la tDCS?, ¿cuál de estas terapias es más efectiva? Estos interrogantes surgen de resultados contradictorios encontrados en algunas investigaciones. Por ejemplo, en el estudio realizado por Elsner et al [30], el tamaño del efecto fue pequeño (diferencia de medias estandarizada $=0,31$ ). El estudio de Otal et al [31], también informó de efectos no significativos con la C-tDCS (diferencia de medias estandarizada $=0,42$ ). Es decir, el tamaño del efecto después de la estimulación con tDCS a menudo fue pequeño y experimentó mucha variabilidad [14,30]. En una revisión Cochrane [16] se llegó a la conclusión de que el uso de la tDCS para mejorar las funciones del lenguaje en personas con afasia no es efectivo. Sin embargo, hay autores que afirman que estos resultados deben interpretarse con cautela, ya que los estudios incluidos en la revisión de Elsner et al [16] difirieron en cuanto al tipo y la gravedad de la afasia, así como en los parámetros de la estimulación [14]. Por su parte, Lefaucheur et al [32] afirman que el nivel de evidencia de la tDCS en la afasia no es suficiente para garantizar la eficacia o el potencial terapéutico de ninguno de los protocolos de rehabilitación de personas con afasia después de un ictus. Sin embargo, una revisión reciente sugiere que las sesiones repetidas de tDCS combinadas con terapia de lenguaje podrían 
mejorar la rehabilitación en la afasia crónica postictus [33]. Asimismo, en una revisión Cochrane más reciente [34], se concluye que actualmente no existen pruebas de que la tDCS sea mejor en el grupo de intervención que en el grupo control cuando se analiza la intervención en la comunicación funcional. Sin embargo, existen pruebas limitadas respecto a que la tDCS puede mejorar el rendimiento de la denominación de sustantivos, pero no de verbos.

Respecto a la recuperación de la anomia, algunas revisiones sistemáticas disponibles concluyen que no hay pruebas de eficacia de la tDCS en la rehabilitación de la anomia [16,35]; por el contrario, un metaanálisis publicado en 2018 concluye que sesiones repetitivas de tDCS pueden mejorar la anomia [36]. Otra revisión sistemática de 2015 sugiere que la tDCS resulta efectiva, a pesar de la variedad de parámetros de estimulación, las características de las personas con afasia y los tratamientos logopédicos utilizados en los diversos estudios [21]. Así pues, se requieren más estudios para alcanzar un consenso urgente, ya que estos resultados ambiguos obstaculizan la implementación de programas de intervención.

\section{Pacientes y métodos}

\section{Criterios de inclusión}

Se consideraron estudios controlados aleatorizados, los cuales incluían a personas adultas de ambos sexos con afasia aguda, postaguda o de carácter crónico, estudios que realizaban intervenciones online (aplicación de tDCS conjuntamente con cualquier tipo de terapia de la afasia), así como intervenciones offline (realización de entrenamiento logopédico antes o después de la tDCS). Asimismo, los estudios incluidos abarcan un período de 10 años (1 de enero de 2009 hasta 31 de enero de 2019). Se han incluido publicaciones en inglés y en castellano.

\section{Estrategia de búsqueda}

Se recurrió a tres bases de datos PubMed, Scopus y Embase. Las palabras clave utilizadas en la búsqueda fueron las siguientes: 'transcranial direct current stimulation and aphasia', 'tDCS and aphasia,' 'cortical modulation and language recovery', ' $t D C S$ and language recovery', 'electrical stimulation therapy and aphasia rehabilitation', 'sham transcranial direct current stimulation and aphasia', 'anodal transcranial direct current stimulation and aphasia', 'cathodal transcranial direct current stimula-
Figura. Diagrama de flujo que refleja el proceso de selección y exclusión de artículos, de acuerdo con los criterios PRISMA [37].

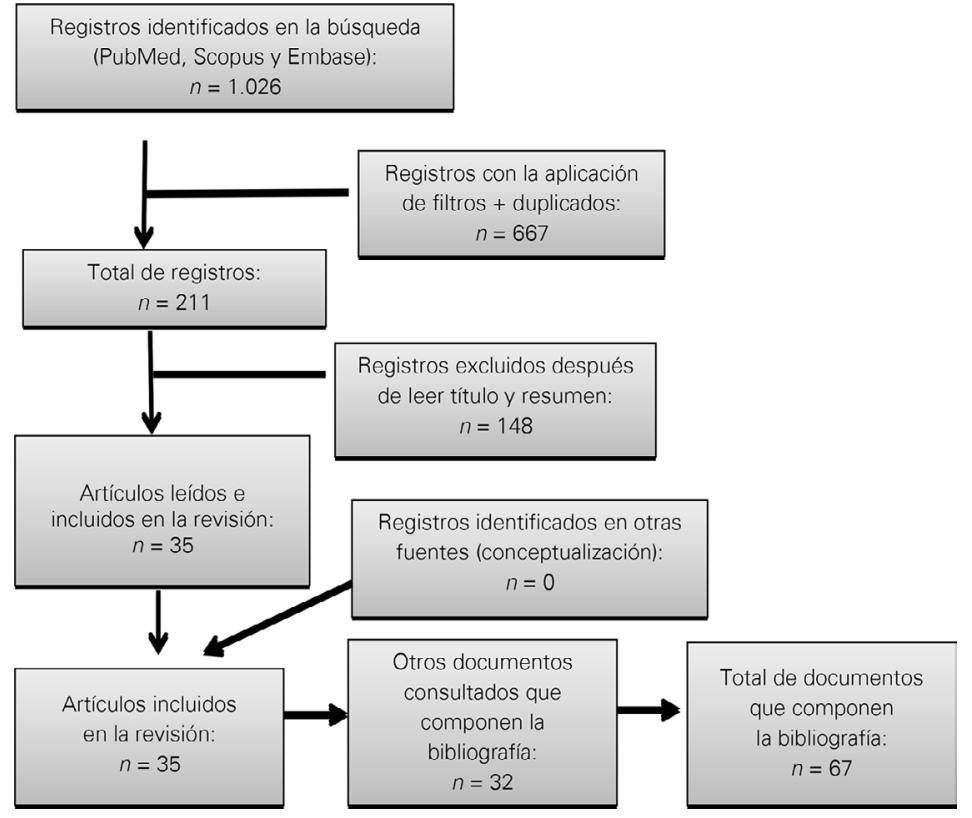

tion', 'non invasive and brain and stimulation and aphasia', 'neuromodulation and aphasia' y 'neurostimulation and aphasia'. Estas palabras se combinaron con los operadores booleanos 'AND' y 'OR' (Figura) [37].

\section{Resultados}

\section{Organización cerebral, sitio y modo de acción de la tDCS}

Anglade et al [38] sugieren una hipótesis basándose en la teoría de la desinhibición transcallosa, según la cual, la red lingüística del hemisferio derecho es menos eficiente que la del izquierdo en un sujeto sano y en caso de una lesión cerebral. La hipótesis sugiere que la desinhibición del hemisferio derecho puede limitar la recuperación cuando algunas de las áreas del lenguaje del lado izquierdo mantienen las funciones casi intactas. Por ello, la estimulación inhibitoria del hemisferio derecho estaría indicada en este caso. Por otro lado, en caso de lesiones extensas del hemisferio izquierdo y cuando hay una mala función del lenguaje residual, la inhibición del hemisferio izquierdo sería potencialmente contra- 
producente. Según los autores, solo en ese caso se puede esperar que la estimulación excitatoria del hemisferio derecho muestre algún beneficio. En este sentido, varios estudios han demostrado que, cuando se produce un daño extenso en el hemisferio izquierdo, se puede buscar una activación del hemisferio derecho, dado que este hemisferio puede desarrollar un papel compensatorio, aunque muy parcial, así como mejorar la precisión en tareas de lenguaje [39]. En esta línea, Winhuisen et al [40] argumentan que la recuperación de la red lingüística del hemisferio izquierdo es más efectiva para la recuperación del lenguaje después de un ictus, pero, en algunos casos, el hemisferio derecho se integra con éxito. En todo caso, estaríamos hablando de que, cuando se producen lesiones pequeñas, con preservación de algunas áreas del hemisferio dominante, la recuperación puede depender del reclutamiento de áreas del lenguaje residuales junto con un aumento de la actividad perilesional. Por el contrario, en caso de lesiones grandes, que incluyen áreas del lenguaje primario, la recuperación puede depender del reclutamiento de áreas del lenguaje homotópicas no dominantes [9]. Por otro lado, Hartwigsen [14] propone un modelo para promover la recuperación del lenguaje después de un ictus. Este modelo asume la contribución de las regiones del hemisferio izquierdo y derecho en la recuperación del lenguaje en diferentes momentos. En ese mismo sentido, Saur et al [41] sugieren que, después de un ictus, cuando hay daño en el hemisferio izquierdo y no ha transcurrido tiempo suficiente para reclutar regiones homotópicas en el hemisferio derecho, ninguno de los dos hemisferios responde para el procesamiento del lenguaje. Esto es consistente con la observación de que los déficits de lenguaje de los pacientes son más graves durante la fase aguda [42]. En la fase subaguda, las áreas homotópicas del lado derecho parecen asumir algunas funciones lingüísticas, aunque estas regiones no parecen ser tan eficientes como las áreas del hemisferio izquierdo; por ello, la recuperación del lenguaje de estas regiones generalmente se asocia con una recuperación del lenguaje más limitada [9]. Por último, con el tiempo, el lado izquierdo puede recuperase hasta el punto de que los centros del lenguaje del hemisferio izquierdo pueden realizar dichas funciones [41]. Estos cambios dinámicos en la actividad cerebral asociada con el lenguaje podrían, en teoría, informar qué enfoque de estimulación cerebral es el más útil de acuerdo con la etapa de recuperación del ictus [43]. Teniendo en cuenta la evidencia que respalda los modelos de recuperación de la afasia en los hemisferios izquierdo y derecho, lo más probable es que el proceso sea dinámico e implique cambios neuroplásticos en los dos hemisferios [41,43]. La organización del lenguaje es un proceso dinámico y la participación de diversos mecanismos es importante en diferentes grados y distintos momentos después del ictus [43].

\section{tDCS según la evolución de la afasia. Etapas aguda, subaguda o crónica}

Según Marsh y Hillis [44], la recuperación del lenguaje después de un ictus tiene lugar durante tres etapas temporales superpuestas: aguda (horas a días), subaguda (semanas a meses) y crónica (meses a años). Según Fregni et al [45], la fecha de inicio de la estimulación es una variable importante por dos razones: en primer lugar, porque la selección de participantes en la etapa aguda podría dificultar la observación de los efectos de la tDCS, ya que sería difícil saber qué factores influyen en la recuperación, dado que los resultados de la estimulación podrían deberse a la recuperación espontánea de la afasia o los efectos de la tDCS. En segundo lugar, la C-tDCS se considera como un tratamiento que potencialmente podría provocar convulsiones epilépticas después de un ictus. En este sentido, Burn et al [46] afirman que el riesgo de convulsiones es mayor durante el primer año posterior al accidente cerebrovascular; asimismo, es más probable que se produzcan convulsiones cuando las lesiones son grandes en zonas anteriores y si la etiología es isquémica. Por ello, De Aguiar et al [21] aconsejan evitar el uso de la tDCS en esta ventana de tiempo y en personas con afasia que presentan estas características. En todo caso, la falta de criterios estrictos de seguridad, especialmente con personas que están en una etapa aguda, limita que el uso de la tDCS pueda extenderse [21]. Esa limitación ha influido en que existan pocos estudios en personas con afasia en esa etapa [42].

\section{Estudios que realizaron la estimulación en la etapa aguda}

Sólo se encontró un estudio que realizó tDCS en esta etapa. En este sentido, Polanowska et al [47] llevaron a cabo un estudio con personas con afasia en la etapa aguda, de cuyos resultados se desprende que no hay pruebas suficientemente sólidas para respaldar la A-tDCS sobre el área de Broca como un tratamiento adyuvante para la rehabilitación de la afasia no fluente. Una observación que se puede hacer a este estudio es que realizaron la tDCS sin terapia logopédica coadyuvante, lo que puede haber influido en los resultados (Tabla) [48-79]. 
Tabla. Estimulación de corriente continua transcraneal (tDCS) e intervención logopédica en personas con afasia: revisión sistemática.

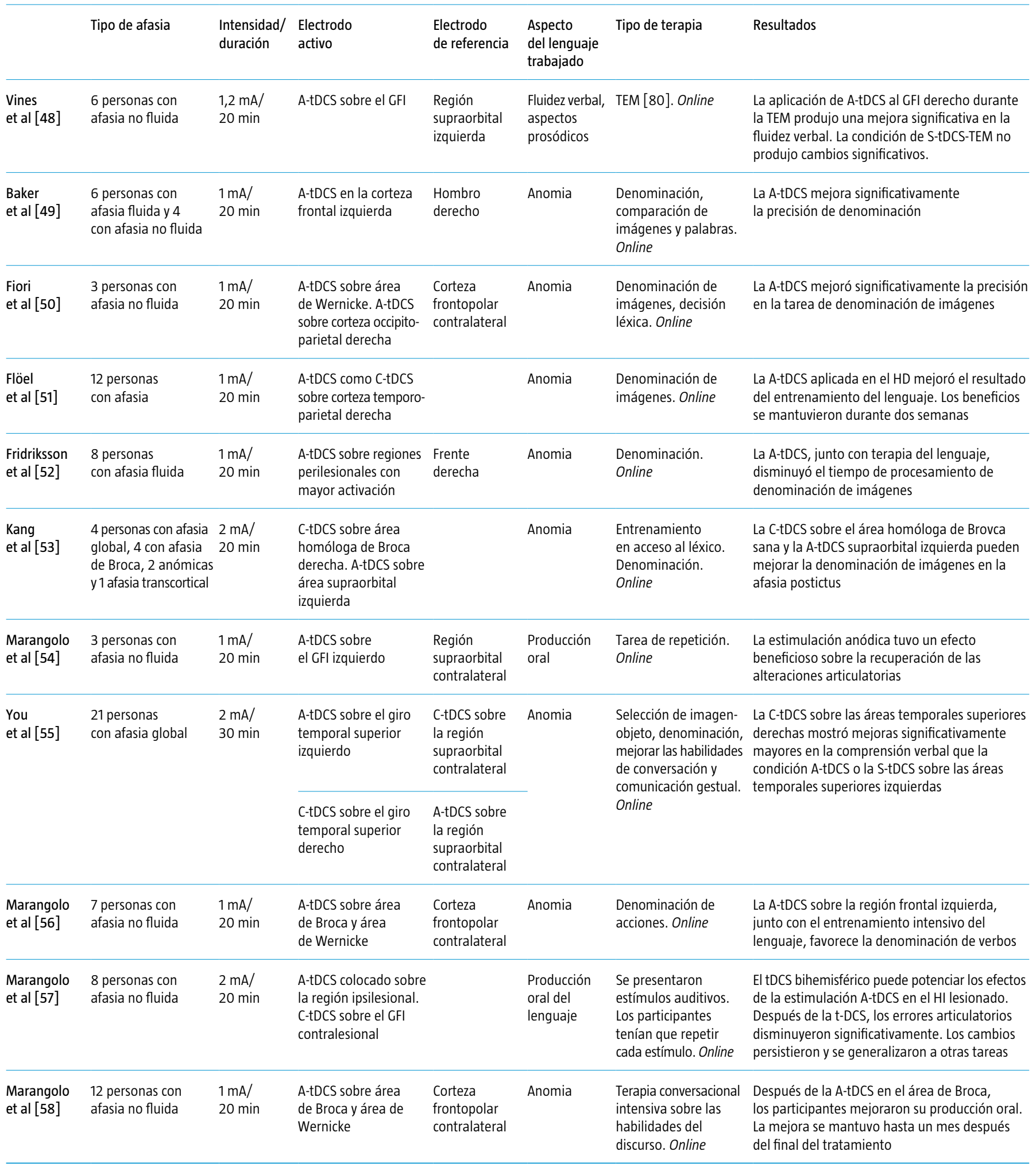


Tabla. Estimulación de corriente continua transcraneal (tDCS) e intervención logopédica en personas con afasia: revisión sistemática (cont.).

\begin{tabular}{|c|c|c|c|c|c|c|c|}
\hline & $\begin{array}{l}\text { Tipo } \\
\text { de afasia }\end{array}$ & $\begin{array}{l}\text { Intensidad/ } \\
\text { duración }\end{array}$ & $\begin{array}{l}\text { Electrodo } \\
\text { activo }\end{array}$ & $\begin{array}{l}\text { Electrodo } \\
\text { de referencia }\end{array}$ & $\begin{array}{l}\text { Aspecto } \\
\text { del lenguaje } \\
\text { trabajado }\end{array}$ & $\begin{array}{l}\text { Tipo } \\
\text { de terapia }\end{array}$ & Resultados \\
\hline
\end{tabular}

\begin{tabular}{|c|c|c|c|c|c|c|c|}
\hline $\begin{array}{l}\text { Lee } \\
\text { et al [60] }\end{array}$ & $\begin{array}{l}6 \text { personas con } \\
\text { afasia no fluida y } \\
5 \text { con afasia fluida }\end{array}$ & $\begin{array}{l}2 \mathrm{~mA} / \\
30 \mathrm{~min}\end{array}$ & A-tDCS sobre el CFI & $\begin{array}{l}\text { Músculo } \\
\text { buccinador } \\
\text { derecho }\end{array}$ & Anomia & $\begin{array}{l}\text { Denominación y } \\
\text { lectura de párrafos } \\
\text { cortos, durante los } \\
\text { últimos } 15 \text { de tDCS. } \\
\text { Online }\end{array}$ & $\begin{array}{l}\text { La A-tDCS sobre el GFI izquierdo y la C-tDCS } \\
\text { sobre el GFI derecho pueden ser más efectivas } \\
\text { que solo la A-tDCS sobre el GFI izquierdo. } \\
\text { El tiempo de respuesta se acorto solo después } \\
\text { del montaje doble de tDCS }\end{array}$ \\
\hline
\end{tabular}

\begin{tabular}{|c|c|c|c|c|c|c|c|}
\hline $\begin{array}{l}\text { Polanowska } \\
\text { et al [61] }\end{array}$ & $\begin{array}{l}13 \text { personas con } \\
\text { afasia fluida y } 24 \\
\text { con afasia no fluida }\end{array}$ & $\begin{array}{l}1 \mathrm{~mA} / \\
10 \mathrm{~min}\end{array}$ & $\begin{array}{l}\text { A-tDCS sobre } \\
\text { área de Broca }\end{array}$ & $\begin{array}{l}\text { Área } \\
\text { supraorbital } \\
\text { derecha }\end{array}$ & Anomia & $\begin{array}{l}\text { Comprensión verbal, } \\
\text { denominación, } \\
\text { repetición de } \\
\text { palabras u oraciones, } \\
\text { construcción de } \\
\text { oraciones, lectura } \\
\text { y escritura. Online }\end{array}$ & $\begin{array}{l}\text { Los hallazgos no apoyan los beneficios funcionales } \\
\text { de la A-tDCS durante la rehabilitación temprana de } \\
\text { la afasia posterior al ictus }\end{array}$ \\
\hline
\end{tabular}

\begin{tabular}{|c|c|c|c|c|c|c|c|}
\hline $\begin{array}{l}\text { Polanowska } \\
\text { et al [47] }\end{array}$ & $\begin{array}{l}24 \text { personas con } \\
\text { afasia no fluida }\end{array}$ & $\begin{array}{l}1 \mathrm{~mA} / \\
10 \mathrm{~min}\end{array}$ & $\begin{array}{l}\text { A-tDCS sobre } \\
\text { área de Broca }\end{array}$ & $\begin{array}{l}\text { A-tDCS } \\
\text { sobre área } \\
\text { supraorbital } \\
\text { derecha }\end{array}$ & Anomia & $\begin{array}{l}\text { Denominación. } \\
\text { Online }\end{array}$ & $\begin{array}{l}\text { Según el estudio, no hay pruebas suficientemente } \\
\text { sólidas para respaldar la A-tDCS repetida sobre } \\
\text { el área de Broca del HI lesionado como un } \\
\text { tratamiento adyuvante para la rehabilitación } \\
\text { de la afasia no fluida en las etapas agudas } \\
\text { posteriores al ictus }\end{array}$ \\
\hline
\end{tabular}

\begin{tabular}{|c|c|c|c|c|c|c|c|}
\hline $\begin{array}{l}\text { Santos } \\
\text { et al [62] }\end{array}$ & $\begin{array}{l}4 \text { personas con afasia } \\
\text { mixta, } 8 \text { con afasia } \\
\text { de Broca y } 7 \text { con } \\
\text { afasia anómica }\end{array}$ & $\begin{array}{l}2 \mathrm{~mA} / \\
20 \mathrm{~min}\end{array}$ & $\begin{array}{l}\text { A-tDCS sobre } \\
\text { área supraorbital. } \\
\text { C-tDCS sobre corteza } \\
\text { motora contralateral }\end{array}$ & & Anomia & $\begin{array}{l}\text { Denominación. } \\
\text { Online }\end{array}$ & $\begin{array}{l}\text { La C-tDCS en la corteza motora primaria no } \\
\text { lesionada se asocia con mejoras significativas } \\
\text { en comprensión de frases simples, denominación } \\
\text { y fluidez verbal de nombres de animales }\end{array}$ \\
\hline $\begin{array}{l}\text { Volpato } \\
\text { et al [63] }\end{array}$ & $\begin{array}{l}8 \text { personas } \\
\text { con afasia }\end{array}$ & $\begin{array}{l}2 \mathrm{~mA} / \\
20 \mathrm{~min}\end{array}$ & $\begin{array}{l}\text { A-tDCS sobre } \\
\text { área de Broca }\end{array}$ & $\begin{array}{l}\text { Área } \\
\text { supraorbital } \\
\text { contralateral }\end{array}$ & Anomia & $\begin{array}{l}\text { Denominación, } \\
\text { producción del } \\
\text { lenguaje y ejercicios } \\
\text { de comprensión. } \\
\text { Online }\end{array}$ & $\begin{array}{l}\text { No hay diferencias significativas entre el A-tDCS } \\
\text { o sham tanto para tareas de denominación de } \\
\text { objetos como de acciones. Los resultados del } \\
\text { estudio indican que el enfoque de tDCS online no } \\
\text { es eficaz en el tratamiento de la afasia crónica }\end{array}$ \\
\hline $\begin{array}{l}\text { Marangolo } \\
\text { et al [64] }\end{array}$ & $\begin{array}{l}8 \text { personas con } \\
\text { afasia no fluida }\end{array}$ & $\begin{array}{l}1 \mathrm{~mA} / \\
20 \mathrm{~min}\end{array}$ & $\begin{array}{l}\text { A-tDCS sobre área } \\
\text { de Broca y A-tDCS } \\
\text { sobre área de } \\
\text { Wernicke }\end{array}$ & $\begin{array}{l}\text { Corteza } \\
\text { frontotemporal } \\
\text { contralateral }\end{array}$ & $\begin{array}{l}\text { Trastornos } \\
\text { productivos } \\
\text { del lenguaje }\end{array}$ & $\begin{array}{l}\text { Terapia de la } \\
\text { conversación. } \\
\text { Online }\end{array}$ & $\begin{array}{l}\text { Tras el tratamiento, los participantes produjeron } \\
\text { descripciones con un mayor número de palabras } \\
\text { y, lo más importante, un uso más preciso de } \\
\text { los dispositivos de cohesión. La estimulación } \\
\text { sobre el GFI izquierdo produjo una mejora en } \\
\text { la capacidad de producir un discurso cohesivo }\end{array}$ \\
\hline $\begin{array}{l}\text { Vestito } \\
\text { et al [66] }\end{array}$ & $\begin{array}{l}3 \text { personas } \\
\text { con afasia }\end{array}$ & $\begin{array}{l}1,5 \mathrm{~mA} / \\
20 \mathrm{~min}\end{array}$ & $\begin{array}{l}\text { A-tDCS sobre la región } \\
\text { frontal izquierda }\end{array}$ & $\begin{array}{l}\text { Región } \\
\text { supraorbital } \\
\text { contralateral }\end{array}$ & Anomia & $\begin{array}{l}\text { Denominación de } \\
\text { imágenes. Online }\end{array}$ & $\begin{array}{l}\text { La A-tDCS produjo una mejora significativa en } \\
\text { la denominación de nombres en relación con } \\
\text { la condición inicial y simulada. Esta mejora se } \\
\text { mantuvo unas } 16 \text { semanas después del tratamiento }\end{array}$ \\
\hline
\end{tabular}


Tabla. Estimulación de corriente continua transcraneal (tDCS) e intervención logopédica en personas con afasia: revisión sistemática (cont.).

\begin{tabular}{|c|c|c|c|c|c|c|c|}
\hline & $\begin{array}{l}\text { Tipo } \\
\text { de afasia }\end{array}$ & $\begin{array}{l}\text { Intensidad/ } \\
\text { duración }\end{array}$ & $\begin{array}{l}\text { Electrodo } \\
\text { activo }\end{array}$ & $\begin{array}{l}\text { Electrodo } \\
\text { de referencia }\end{array}$ & $\begin{array}{l}\text { Aspecto } \\
\text { del lenguaje } \\
\text { trabajado }\end{array}$ & $\begin{array}{l}\text { Tipo } \\
\text { de terapia }\end{array}$ & Resultados \\
\hline $\begin{array}{l}\text { De Aguiar } \\
\text { et al [21] }\end{array}$ & $\begin{array}{l}9 \text { personas } \\
\text { con afasia }\end{array}$ & $\begin{array}{l}1 \mathrm{~mA} / \\
20 \mathrm{~min}\end{array}$ & $\begin{array}{l}\text { A-tDCS sobre el área } \\
\text { perilesional izquierda. } \\
\text { C-tDCS sobre el } \\
\text { homologo derecho } \\
\text { del área de Broca o } \\
\text { el área de Wernicke }\end{array}$ & & Anomia & $\begin{array}{l}\text { Protocolo ACTION } \\
\text { [83]. Denominación de } \\
\text { acciones, finalización } \\
\text { de oraciones con } \\
\text { infinitivo. Online }\end{array}$ & $\begin{array}{l}\text { Mejor producción de verbos tratados y no tratados, } \\
\text { lo que demuestra la eficacia del tratamiento } \\
\text { logopédico combinado con tDCS y su potencial } \\
\text { para producir generalización }\end{array}$ \\
\hline $\begin{array}{l}\text { Campana } \\
\text { et al [67] }\end{array}$ & $\begin{array}{l}20 \text { personas con } \\
\text { afasia no fluida }\end{array}$ & $\begin{array}{l}2 \mathrm{~mA} / \\
20 \mathrm{~min}\end{array}$ & $\begin{array}{l}\text { A-tDCS colocado } \\
\text { sobre F5 del sistema } \\
\text { internacional } 10-20\end{array}$ & $\begin{array}{l}\text { Corteza } \\
\text { frontopolar } \\
\text { contralateral }\end{array}$ & Anomia & $\begin{array}{l}\text { Descripción de imágenes, } \\
\text { denominación de } \\
\text { sustantivos y verbos. } \\
\text { Online }\end{array}$ & $\begin{array}{l}\text { Mejora significativa en el rendimiento para } \\
\text { las tres subpruebas de lenguaje, cuando el } \\
\text { tratamiento se asoció con la A-tDCS }\end{array}$ \\
\hline $\begin{array}{l}\text { Cipollari } \\
\text { et al [68] }\end{array}$ & $\begin{array}{l}6 \text { personas con } \\
\text { afasia no fluida }\end{array}$ & $\begin{array}{l}2 \mathrm{~mA} / \\
20 \mathrm{~min}\end{array}$ & $\begin{array}{l}\text { A-tDCS sobre } \\
\text { el GFI derecho }\end{array}$ & $\begin{array}{l}\text { Corteza } \\
\text { frontopolar } \\
\text { contralateral }\end{array}$ & Apraxia & $\begin{array}{l}\text { TEM para tratar la } \\
\text { apraxia del habla. } \\
\text { Online }\end{array}$ & $\begin{array}{l}\text { Mejora significativa en la precisión de la } \\
\text { respuesta, tanto para las condiciones anódicas } \\
\text { como simuladas. La mejora se mantuvo una } \\
\text { semana después del tratamiento }\end{array}$ \\
\hline $\begin{array}{l}\text { Shah-Basak } \\
\text { et al [69] }\end{array}$ & $\begin{array}{l}12 \text { personas con } \\
\text { afasia no fluida }\end{array}$ & $\begin{array}{l}2 \mathrm{~mA} / \\
20 \mathrm{~min}\end{array}$ & $\begin{array}{l}\text { Ánodo F3, cátodo F3, } \\
\text { ánodo F4 y cátodo F4 }\end{array}$ & $\begin{array}{l}\text { Mastoideo } \\
\text { contralateral }\end{array}$ & Anomia & $\begin{array}{l}\text { Terapia inducida por } \\
\text { restricciones [86]. } \\
\text { Online }\end{array}$ & $\begin{array}{l}\text { El montaje más consistente para provocar una } \\
\text { mejora transitoria fue la C-tDCS del lóbulo frontal } \\
\text { izquierdo. La mejora persistió durante dos meses }\end{array}$ \\
\hline $\begin{array}{l}\text { Wu } \\
\text { et al [70] }\end{array}$ & $\begin{array}{l}8 \text { personas con afasia } \\
\text { de Broca, } 2 \text { con afasia } \\
\text { mixta, } 1 \text { con afasia } \\
\text { anómica y } 1 \text { con } \\
\text { afasia de conducción }\end{array}$ & $\begin{array}{l}1,2 \mathrm{~mA} / \\
20 \mathrm{~min}\end{array}$ & $\begin{array}{l}\text { A-tDCS sobre la } \\
\text { región perisilviana } \\
\text { posterior izquierda }\end{array}$ & $\begin{array}{l}\text { Hombro } \\
\text { no afectado }\end{array}$ & Anomia & $\begin{array}{l}\text { Denominación. } \\
\text { Online }\end{array}$ & $\begin{array}{l}\text { La A-tDCS sobre la región perisilviana posterior } \\
\text { izquierda, junto con la terapia del lenguaje, en } \\
\text { afasias postagudas, puede mejorar la denominación } \\
\text { y la identificación auditiva palabra-imagen y } \\
\text { aumentar la excitabilidad cortical en la red } \\
\text { lingüística }\end{array}$ \\
\hline $\begin{array}{l}\text { Lifshitz } \\
\text { Ben Basat } \\
\text { et al [71] }\end{array}$ & $\begin{array}{l}2 \text { personas con afasia } \\
\text { de Broca y } 5 \text { con } \\
\text { afasia anómica }\end{array}$ & $\begin{array}{l}2 \mathrm{~mA} / \\
10 \mathrm{~min}\end{array}$ & $\begin{array}{l}\text { A-tDCS sobre el GFI } \\
\text { izquierdo y derecho } \\
\text { y el GTS izquierdo y } \\
\text { derecho. C-tDCS sobre } \\
\text { las mismas ubicaciones }\end{array}$ & $\begin{array}{l}\text { Área } \\
\text { supraorbital } \\
\text { contralateral }\end{array}$ & Anomia & $\begin{array}{l}\text { Denominación de } \\
\text { imágenes. Online }\end{array}$ & $\begin{array}{l}\text { Seis sesiones de tDCS mejoraron significativamente } \\
\text { la denominación en personas con afasia crónica, } \\
\text { mientras que la estimulación simulada no tuvo } \\
\text { tal efecto. La mejora se mantuvo tres meses }\end{array}$ \\
\hline $\begin{array}{l}\text { Marangolo } \\
\text { et al [72] }\end{array}$ & $\begin{array}{l}9 \text { personas con } \\
\text { afasia no fluida }\end{array}$ & $\begin{array}{l}2 \mathrm{~mA} / \\
20 \mathrm{~min}\end{array}$ & $\begin{array}{l}\text { A-tDCS sobre el área } \\
\text { de Broca y C-tDCS sobre } \\
\text { el homólogo derecho }\end{array}$ & & $\begin{array}{l}\text { Trastornos } \\
\text { articulatorios }\end{array}$ & $\begin{array}{l}\text { Repetición de } \\
\text { estímulos auditivos. } \\
\text { Online }\end{array}$ & $\begin{array}{l}\text { Después de sham, los cambios de conectividad } \\
\text { se limitaron al HD, la estimulación t-DCS dio } \\
\text { lugar a un aumento de la conectividad más fuerte } \\
\text { en el HI. La tDCS bilateral determina los cambios } \\
\text { de conectividad funcional en el hemisferio } \\
\text { lesionado, lo que mejora el proceso de recuperación } \\
\text { del lenguaje en pacientes con ictus }\end{array}$ \\
\hline $\begin{array}{l}\text { Meinzer } \\
\text { et al [73] }\end{array}$ & $\begin{array}{l}26 \text { personas } \\
\text { con afasia }\end{array}$ & $\begin{array}{l}1 \mathrm{~mA} / \\
20 \mathrm{~min}\end{array}$ & $\begin{array}{l}\text { A-tDCS sobre C3 del } \\
\text { sistema EEG } 10-20 \\
\text { izquierdo. C-tDCS sobre } \\
\text { la región supraorbital } \\
\text { derecha }\end{array}$ & & Anomia & $\begin{array}{l}\text { Denominación. } \\
\text { Online }\end{array}$ & $\begin{array}{l}\text { La denominación de nombres mejoró de forma } \\
\text { significativa después de la intervención, tanto } \\
\text { en los grupos de A-tDCS como de S-tDCS, con } \\
\text { una tendencia a mayores ganancias en la A-tDCS. } \\
\text { La mejora se mantuvo seis meses en el grupo } \\
\text { de A-tDCS y obtuvo transferencias a ítems no } \\
\text { entrenados }\end{array}$ \\
\hline $\begin{array}{l}\text { Darkow } \\
\text { et al [74] }\end{array}$ & $\begin{array}{l}16 \text { personas } \\
\text { con afasia }\end{array}$ & $\begin{array}{l}1 \mathrm{~mA} / \\
20 \mathrm{~min}\end{array}$ & $\begin{array}{l}\text { A-tDCS sobre C3 del } \\
\text { sistema EEG } 10-20 \\
\text { izquierdo. C-tDCS sobre } \\
\text { la región supraorbital } \\
\text { derecha }\end{array}$ & & Anomia & $\begin{array}{l}\text { Denominación. } \\
\text { Online }\end{array}$ & $\begin{array}{l}\text { La A-tDCS contribuyó a una mejor } \\
\text { conectividad dentro de la red lingüística }\end{array}$ \\
\hline $\begin{array}{l}\text { Dos Santos } \\
\text { et al [75] }\end{array}$ & $\begin{array}{l}13 \text { personas } \\
\text { con afasia }\end{array}$ & $\begin{array}{l}2 \mathrm{~mA} / \\
20 \mathrm{~min}\end{array}$ & $\begin{array}{l}\text { A-tDCS sobre el área } \\
\text { de Broca. C-tDCS sobre } \\
\text { F8 del sistema EEG }\end{array}$ & & Anomia & $\begin{array}{l}\text { Denominación. } \\
\text { Online }\end{array}$ & $\begin{array}{l}\text { Hubo diferencias estadísticamente significativas } \\
\text { en las tareas de denominación de imágenes } \\
\text { después de una sesión de tDCS y después de } \\
\text { la estimulación sham }\end{array}$ \\
\hline
\end{tabular}


Tabla. Estimulación de corriente continua transcraneal (tDCS) e intervención logopédica en personas con afasia: revisión sistemática (cont.).

\begin{tabular}{|c|c|c|c|c|c|c|c|}
\hline & $\begin{array}{l}\text { Tipo } \\
\text { de afasia }\end{array}$ & $\begin{array}{l}\text { Intensidad/ } \\
\text { duración }\end{array}$ & $\begin{array}{l}\text { Electrodo } \\
\text { activo }\end{array}$ & $\begin{array}{l}\text { Electrodo } \\
\text { de referencia }\end{array}$ & $\begin{array}{l}\text { Aspecto } \\
\text { del lenguaje } \\
\text { trabajado }\end{array}$ & $\begin{array}{l}\text { Tipo de } \\
\text { terapia }\end{array}$ & Resultados \\
\hline $\begin{array}{l}\text { Branscheidt } \\
\text { et al [77] }\end{array}$ & $\begin{array}{l}16 \text { personas } \\
\text { con afasia }\end{array}$ & $\begin{array}{l}2 \mathrm{~mA} / \\
20 \mathrm{~min}\end{array}$ & $\begin{array}{l}\text { A-tDCS sobre la } \\
\text { corteza motora } \\
\text { izquierda }\end{array}$ & $\begin{array}{l}\text { Región } \\
\text { supraorbital } \\
\text { derecha }\end{array}$ & & $\begin{array}{l}\text { Decisión léxica de } \\
\text { verbos, sustantivos } \\
\text { y pseudopalabras. } \\
\text { Online }\end{array}$ & $\begin{array}{l}\text { La A-tDCS en la corteza motora del HI mejora } \\
\text { la precisión general en una tarea de decisión } \\
\text { léxica, en palabras de acción y pseudopalabras } \\
\text { similares a las palabras de acción, mientras que } \\
\text { los sustantivos y las pseudopalabras similares } \\
\text { a los sustantivos no se vieron beneficiados } \\
\text { significativamente por la tDCS }\end{array}$ \\
\hline $\begin{array}{l}\text { Marangolo } \\
\text { et al [78] }\end{array}$ & $\begin{array}{l}12 \text { personas con } \\
\text { afasia no fluida }\end{array}$ & $\begin{array}{l}2 \mathrm{~mA} / \\
20 \mathrm{~min}\end{array}$ & $\begin{array}{l}\text { A-tDCS sobre la } \\
\text { corteza cingulada } \\
\text { anterior }\end{array}$ & $\begin{array}{l}\text { Mejilla } \\
\text { derecha }\end{array}$ & Anomia & $\begin{array}{l}\text { Denominación y } \\
\text { repetición de verbos. } \\
\text { Online }\end{array}$ & $\begin{array}{l}\text { Hubo una mejora significativa después de la } \\
\text { C-tDCS sólo en la tarea de generación de verbos. } \\
\text { No hubo diferencias significativas para la } \\
\text { denominación de los verbos entre las condiciones } \\
\text { de A-tDCS o S-tDCS. Los efectos de mantuvieron } \\
\text { más de una semana }\end{array}$ \\
\hline $\begin{array}{l}\text { Pestalozzi } \\
\text { et al [79] }\end{array}$ & $\begin{array}{l}6 \text { personas con afasia } \\
\text { anómica, } 4 \text { con afasia } \\
\text { de conducción, } 3 \text { con } \\
\text { afasia de Broca y } \\
1 \text { con afasia global }\end{array}$ & $\begin{array}{l}1 \mathrm{~mA} / \\
20 \mathrm{~min}\end{array}$ & $\begin{array}{l}\text { Cátodo en la corteza } \\
\text { cerebelosa derecha }\end{array}$ & $\begin{array}{l}\text { Hombro } \\
\text { derecho en } \\
\text { el músculo } \\
\text { deltoides }\end{array}$ & Anomia & $\begin{array}{l}\text { Denominación de } \\
\text { imágenes, tarea de } \\
\text { fluidez fonémica y } \\
\text { tarea de repetición. } \\
\text { Online y Online }\end{array}$ & $\begin{array}{l}\text { La A-tDCS prefrontal izquierda puede mejorar los } \\
\text { procesos que dependen de la interacción entre } \\
\text { el lenguaje y las funciones de control ejecutivo }\end{array}$ \\
\hline
\end{tabular}

A-tDCS: estimulación de corriente continua transcraneal anódica; C-tDCS: estimulación de corriente continua transcraneal catódica; GFI: giro frontal inferior; GTS: giro temporal superior; HD: hemisferio derecho; HI: hemisferio izquierdo; sham: estimulación placebo; S-tDCS: estimulación de corriente continua transcraneal catódica placebo (sham); TEM: terapia de entonación melódica.

\section{Estudios que realizaron la estimulación en la etapa subaguda}

En esta revisión se analizaron cuatro estudios que incluyeron a personas con afasia en la etapa subaguda $[53,55,61,70]$. Con resultados diferentes, Kang et al [53] y You et al [55] obtuvieron resultados positivos; sin embargo, en el estudio de Polanowska et al [61] no se encontraron diferencias significativas.

\section{Estudios que realizaron la estimulación en la etapa crónica}

En esta revisión, el 91\% de los estudios analizados incluyó a personas con afasia crónica, con resultados contradictorios.

\section{Tipo de afasia}

El 42\% de los estudios de esta revisión incluyó a per- sonas con afasia no fluente $[43,47,48,50,54,56-59,63-$ 65,67-69,78]. En cuanto a las afasias fluentes, sólo el estudio de Fridriksson et al [52] incorporó únicamente a personas con afasia fluente. Por otra parte, el 17\% de los estudios de esta revisión incluyó tanto a personas con afasia fluente como no fluente [49, $60,61,63,73,76]$.

Respecto a las tipologías de las afasias, algunos estudios con personas con distintos tipos de afasia, por ejemplo, el de Fridriksson et al [76], incorporaron a personas con afasia global, transcortical motora, de Wernicke, transcortical sensorial, de conducción, anómica y de Broca. Los resultados de este estudio no produjeron beneficios terapéuticos. Posiblemente, los diferentes tipos de afasia influyeron en los resultados, ya que, además, el tratamiento logopédico fue el mismo para todos los pacientes. 


\section{Déficits afásicos}

El 57\% de los estudios de esta revisión se centra en la anomia $[21,43,47,49,50,52,53,56,59,60,62,65-67,69-$ $71,73-75,78,79]$. Otros estudios han realizado la terapia centrada en otros aspectos deficitarios, propios de la afasia: prosodia del lenguaje [48], producción oral [54,57,58,64], comprensión del lenguaje [55] y trastornos articulatorios [72].

\section{Tipo de tareas que se han realizado en la terapia}

El 63\% de los estudios de esta revisión ha realizado tDCS junto con terapias de denominación $[21,43,47$, 49-52,55,56,59,60,62,65-67,69-71,73-75]. Otros estudios la han hecho con terapias de repetición $[54,57$, $72]$, habilidades discursivas [58,64], asociación palabra-dibujo [76] o decisión léxica [77].

\section{Descripción de las diferentes terapias utilizadas}

Intervención en la comunicación funcional Se encontró sólo un estudio que midió los cambios de la comunicación funcional después de la tDCS [58]. En dicho estudio, las personas con afasia informaron acerca de una mejor capacidad de la comunicación después de la tDCS.

Otro estudio exploró el efecto de la tDCS en la comunicación funcional. En dicho estudio, la intervención de la tDCS, junto con la intervención logopédica, no sólo mejoró las habilidades de denominación, sino también la comunicación funcional [73].

\section{Terapia conversacional}

El objetivo de la terapia conversacional es establecer una conversación natural entre un interlocutor y la persona con afasia, en la que participan ambos interlocutores utilizando los recursos comunicativos disponibles (gestos, dibujos, señales, etc.). Asimismo, este tipo de terapia ayuda a que la persona con afasia sea lo más informativa posible [58]. Algunos estudios han encontrado que, en comparación con la condición sham, los efectos combinados de tDCS y la terapia de la conversación condujeron a la producción de un discurso más informativo [58, 64]. Según el estudio de Marangolo et al [58], la estimulación del área de Broca produjo una mejora en la capacidad de las personas con afasia para producir un discurso informativo. Los participantes aumentaron su capacidad para usar información morfosintáctica relevante e incrementaron el número de oraciones producidas; además, los cambios significativos después de la terapia persistieron un mes y se generalizaron. Estos resultados sugieren que el área de Broca es un epicentro cortical que sirve para la selección de representaciones semánticas apropiadas al contexto. Esta información conceptual desencadena la generación de proposiciones que deben organizarse macrolingüísticamente [58]. Por su parte, Marangolo et al [64], en un grupo de ocho participantes con afasia crónica no fluente, realizaron una investigación con el objetivo de observar los efectos potenciales de la tDCS en la mejora del habla espontánea y la capacidad de usar palabras conectivas para establecer la cohesión entre las expresiones. Según los resultados de este estudio, la administración de A-tDCS sobre el área de Broca ayudó a los participantes a producir descripciones con un mayor número de palabras; asimismo, mejoró el uso de dispositivos de cohesión.

\section{Terapia de entonación melódica}

La terapia de entonación melódica aprovecha los efectos de la rehabilitación del ritmo y la prosodia en pacientes con afasias motoras de tipo Broca de intensidad moderada o grave. Esta técnica se inspiró en la observación clínica de que los pacientes con afasia grave podían cantar las letras de las canciones mejor de lo que podían recitar [80].

En un estudio en el que se empleó la A-tDCS sobre el giro frontal inferior posterior derecho en relación con la terapia de entonación melódica [48], los autores encontraron que la A-tDCS produjo una mejoría significativa en la fluidez del habla. Por su parte, Cipollari et al [68] realizaron un estudio con el objetivo de investigar si la A-tDCS aplicada sobre el giro frontal inferior derecho, en relación con la terapia de entonación melódica, podría mejor la capacidad de articular el habla en un grupo de personas con afasia crónica. Los resultados de este estudio mostraron una mejoría significativa en la precisión de la respuesta, tanto para las condiciones anódicas como simuladas. Sin embargo, el efecto beneficioso de la estimulación anódica resultó evidente porque la precisión de la respuesta fue mayor después de la estimulación. Además, las pruebas de seguimiento revelaron que los efectos de la A-tDCS no habían disminuido una semana después de la finalización de la intervención. Según los autores, estos resultados permiten afirmar que la combinación de tDCS y terapia de entonación melódica puede tener efectos duraderos. Asimismo, los resultados del estudio evidenciaron una transferencia de los efectos del tratamiento no sólo a los estímulos tratados, sino también a los no tratados, en la que los pacientes mostraron una reducción de los errores fonológicos en diferentes tareas de producción oral [68]. 


\section{Terapia de observación de acción}

La recuperación de verbos se debe considerar como un aspecto importante en la rehabilitación de la anomia [64]. Según se desprende de un estudio, el enfoque de observación de la acción mejora la recuperación de los verbos solamente pidiendo a las personas con afasia con déficit de recuperación de verbos que observen las acciones humanas y, además, el tratamiento se generaliza a las palabras no tratadas [81].

Respecto a este tipo de terapia, se realizó una revisión sistemática en donde se sugiere que la estimulación cerebral no invasiva y los enfoques de observación de acciones son herramientas muy prometedoras como enfoques de tratamiento complementario para la recuperación de la afasia [64].

\section{Decisión léxica}

Branscheidt et al [77] realizaron un estudio con el objetivo de averiguar si la A-tDCS mejoraba el acceso al léxico en un grupo de personas con afasia crónica. La tarea de las personas era decidir entre verbos y pseudopalabras (parecidas a verbos), y sustantivos y pseudopalabras (parecidas a sustantivos). Aunque no se observaron efectos específicos de la tDCS en las latencias de decisión léxica, los resultados mostraron que la estimulación anódica en la corteza motora del hemisferio izquierdo mejoró la precisión general en las tareas de decisión léxica. Las decisiones léxicas eran significativamente más precisas en la estimulación anódica para palabras de acción y pseudopalabras de acción similar, mientras que las palabras de objeto (sustantivos) y las pseudopalabras de objeto similar no se vieron afectadas significativamente por la tDCS. Algunos autores afirman que las personas con lesiones frontales tienen dificultades para comprender las palabras de acción, mientras que las lesiones temporales se asocian a un acceso deficiente a los sustantivos [77], aunque hay autores que afirman que el procesamiento del lenguaje se basa en redes de interconexión generalizadas en lugar de circunscritas [82].

\section{Terapia de producción de verbos}

De Aguiar et al [21] realizaron un estudio con el objetivo de probar la eficacia de la versión italiana de ACTION, un protocolo de tratamiento de la rehabilitación de la afasia [83], que incluye cuatro pasos que abordan los diferentes niveles de procesamiento necesarios para producir verbos en oraciones simples y declarativas: nivel léxico (denominación de acciones), nivel sintáctico (completar la oración con un verbo en infinitivo), nivel morfosintáctico (completar la oración con un verbo finito) y construcción de la oración. Después de realizar la terapia ACTION junto con la tDCS, los autores observaron una mejor recuperación de los verbos en la construcción de la oración y no en las otras pruebas de verbo. Esto sugiere que la generalización de los estímulos no sólo depende del entrenamiento en la recuperación del verbo léxico o de la recuperación del conocimiento abstracto de la estructura, sino también de la producción de estructuras basadas en predicados [21]. Además, observaron una mejora significativa tanto para los verbos tratados como para los no tratados.

\section{Duración de la estimulación}

Un 83\% de los estudios incluidos realizó la estimulación durante 20 minutos [21,43,48-59,62-64,66-70, 72-79]; un $8 \%$, durante 10 minutos [47,61,71]; un $3 \%$, durante 15 minutos [65]; y un 6\%, durante 30 minutos $[55,60]$.

\section{Frecuencia de las sesiones}

Se observan diferentes frecuencias de las dosis: una sesión [60], dos sesiones -una de C-tDCS y otra de sham tDCS (estimulación placebo) [65]-, tres sesiones [48], cinco sesiones [50], dos semanas [22,43, $49,52-54,62,66,67,69]$, tres semanas [59] y 20 sesiones [78]. Se observa que lo más habitual es la realización de dos semanas de estimulación.

\section{Período de seguimiento}

Los estudios presentan períodos de seguimiento variables. Fridriksson et al [52] hicieron un seguimiento de tres semanas después del final de la estimulación, mientras que Shah-Basak et al [69] realizaron un seguimiento de ocho semanas después de la estimulación; el más prolongado (21 semanas) fue el de Vestito et al [66]. Solo el 6\% de los estudios efectuó seguimiento tras la interrupción de la estimulación.

\section{Tratamiento online frente a offline}

El 86\% de los estudios incluidos realizó una intervención online (mientras los pacientes recibían la tDCS); el $11 \%$, offline (la intervención y la tDCS se aplicaban en distintos momentos); y el 3\%, una mixta, tanto online como offline. Los estudios que realizaron tDCS offline, es decir, sin tratamiento logopédico, no obtuvieron buenos resultados $[47,62,63]$. Estos hallazgos podrían respaldar la hipótesis de que la tDCS aumentaría el efecto beneficioso de la intervención logopédica, pero no sería recomenda- 
ble para obtener efectos de larga duración en solitario (por sí misma) [63]. En este sentido, De Aguiar et al [21] sugieren que la mejor estrategia es utilizar tDCS junto con terapia logopédica online.

Los efectos de la tDCS se pueden mantener hasta una hora después de la estimulación [84]. Esto puede conllevar que no sólo haya estimulación neuronal prolongada, sino que la estimulación logopédica que se haya realizado conjuntamente se pueda prolongar más allá de la terapia, incluso se podría llegar a hablar de un efecto acumulativo de la tDCS aplicada de forma coadyuvante con terapia logopédica. De hecho, se ha sugerido que, si se realizan sesiones repetidas, hay un mayor efecto de la tDCS, lo que sugiere un efecto dependiente de la dosis [36]. En este sentido, se ha planteado la hipótesis de que los efectos de corta duración inducidos por una sola sesión de tDCS se acumularían con sesiones repetitivas y crearían temporalmente un estado que promovería el reaprendizaje del lenguaje [85]. Es decir, más sesiones ampliarían de manera gradual la ventana terapéutica de los procesos de consolidación en los que la terapia logopédica, realizada junto con la tDCS, reforzaría las redes que se facilitan mediante neuromodulación con la tDCS [86].

\section{Eficacia de la tDCS en la afasia}

En esta revisión sistemática, el $89 \%$ de los estudios respalda la eficacia de la tDCS en el tratamiento de la afasia [21,43,48-60,62,64-66,67-75,77-79]. En dichos estudios se obtienen resultados que ayudan a la recuperación de la afasia independientemente del tipo de terapia, del tipo de afasia, de los montajes (tanto anódico como catódico) y de las áreas cerebrales estimuladas. El denominador común de estos estudios es que utilizan la tDCS con terapia logopédica, aunque se tiene que matizar que, en el estudio de Fridriksson et al [76], pese a realizar intervención online, sólo se observaron mejoras en las tareas de denominación, y los resultados fueron estadísticamente no significativos en el resto de las variables dependientes. Este estudio representa el $2 \%$ de los revisados. Por otro lado, sólo un $9 \%$ de los estudios no obtuvo resultados que respalden el uso de la tDCS en la afasia $[47,61,65]$, y estos estudios se realizaron offline. Estos resultados sugieren la importancia de realizar intervenciones de tDCS junto con la terapia logopédica.

\section{Discusión}

Esta revisión sistemática tiene como objetivo ofre- cer una visión general sobre la tDCS en personas con afasia desde una visión logopédica. Después de realizar un análisis, se ha llegado a las siguientes conclusiones. La mayoría de los estudios incluye a personas con afasia no fluente; sin embargo, sería necesario realizar intervenciones en personas con afasia fluente para determinar el potencial terapéutico de la tDCS en este tipo de afasias. Por otra parte, la mayoría de los estudios incluye muestras heterogéneas sin tener en cuenta la sintomatología propia de cada tipo de afasia y sin individualizar los tratamientos. Esto puede influir en que no se distingan realmente los beneficios y las limitaciones de la tDCS en la afasia.

La anomia (la dificultad de evocar palabras o de reproducir los sonidos que las componen) es el déficit que más se abordado. Dicho déficit se puede encontrar en el lenguaje oral y escrito, así como en tareas de lenguaje o en el uso espontáneo del habla [87]. La anomia es el trastorno del lenguaje más frecuente y, además, está presente en todos los tipos de afasia [88], pero no se presenta de la misma manera. Según Cuetos et al [89], existen diferentes tipos de anomia en función de cuál sea el mecanismo cognitivo que falla en cada paciente; así pues, según este autor, hay cuatro tipos de anomia: anomia semántica, donde hay trastornos semánticos, pero no fonológicos; anomia fonológica, donde hay errores en las tareas fonológicas, pero se conserva la semántica; anomia pura, en donde no se observan errores en las tareas fonológicas ni semánticas, pero se tienen graves dificultades en las tareas de denominación; y anomia mixta, donde hay errores en las tareas fonológicas y en las semánticas. En esta revisión se observa que los diferentes estudios no hacen caso de la tipología de la anomia. En este sentido, estamos de acuerdo con De Aguiar et al [21] cuando sugieren que no es muy recomendable usar solo un tipo de tratamiento para todos los distintos tipos de anomia, ya que la visión coadyuvante de la tDCS en la logopedia puede ser errónea. Se debe hacer un esfuerzo para comprender si la técnica es igual de efectiva al tratar el daño desde diferentes mecanismos del lenguaje, por ejemplo, la anomia 'semántica' frente a la 'fonológica' [21]. En consecuencia, la rehabilitación de la anomia debería diseñarse a partir de una adecuada clasificación y partiendo del conocimiento de las características de cada paciente y de su contexto, con la finalidad de realizar una rehabilitación individualizada, adaptada y eficaz.

Otra conclusión importante es que es mejor realizar la tDCS junto con terapia logopédica online. La tDCS no debería sustituir a la terapia logopédica, sino complementarla [90]. 
En el 89\% de los estudios incluidos en esta revisión se obtienen resultados que ayudan a la recuperación de la afasia, independientemente del tipo de terapia, del tipo de afasia, de los montajes y de las áreas cerebrales estimuladas. La tDCS es una técnica que puede ayudar a superar los déficits del lenguaje de las personas con afasia. Los resultados pueden ser más alentadores si, a su vez, se realizan intervenciones logopédicas de calidad, es decir, utilizando programas y ejercicios que hayan surgido de una práctica basada en la evidencia; además, si se individualiza la terapia y se busca que ésta tenga una repercusión en la vida cotidiana de la persona con afasia, se puede conseguir que la persona logre una comunicación plena con su entorno.

Bibliografía

1. Terradillos E, López-Higes R. Guía de intervención logopédica en las afasias. Madrid: Síntesis; 2016.

2. Laska AC, Hellblom A, Murray V, Kahan T, Von Arbin M Aphasia in acute stroke and relation to outcome. J Intern Med 2011; 249: 413-22.

3. Berthier ML. Poststroke aphasia: epidemiology, pathophysiology and treatment. Drugs Aging 2005; 22: 163-82.

4. Nickels L. Improving word finding: practice makes (closer to) perfect? Aphasiology 2002; 16: 1047-60.

5. Kiran S. What is the nature of poststroke language recovery and reorganization? ISRN Neurol 2012; 2012: 786872.

6. Saur D, Kreher BW, Schnell S, Kümmerer D, Kellmeyer P, Vry MS, et al. Ventral and dorsal pathways for language. Proc Natl Acad Sci U S A 2008; 105: 18035-40.

7. Murdoch BE. Subcortical brain mechanisms in speech and language. Folia Phoniatr Logop 2001; 53: 233-51.

8. Hamilton RH, Chrysikou EG, Coslett B. Mechanisms of aphasia recovery after stroke and the role of noninvasive brain stimulation. Brain Lang 2011; 118: 40-50.

9. Heiss $\mathrm{W}$, Thiel A. A proposed regional hierarchy in recovery of post-stroke aphasia. Brain Lang 2006; 98: 118-23.

10. Thiel A, Habedank B, Herholz K, Kessler J, Winhuisen L, Haupt WF, et al. From the left to the right: How the brain compensates progressive loss of language function. Brain Lang 2006; 98: 57-65.

11. Murase N, Duque J, Mazzocchio R, Cohen LG. Influence of interhemispheric interactions on motor function in chronic stroke. Ann Neurol 2004; 55: 400-9.

12. Chrysikou EG, Hamilton RH. Noninvasive brain stimulation in the treatment of aphasia: exploring interhemispheric relationships and their implications for neurorehabilitation. Restor Neurol Neurosci 2011; 29: 375-94.

13. Saur D, Hartwigsen G. Neurobiology of language recovery after stroke: lessons from neuroimaging studies. Arch Phys Med Rehabil 2012; 93 (Suppl 1): S15-25.

14. Hartwigsen G. Adaptive plasticity in the healthy language network: implications for language recovery after stroke. Neural Plast 2016; 2016: 9674790.

15. Shah PP, Szaflarski JP, Allendorfer J, Hamilton RH. Induction of neuroplasticity and recovery in post-stroke aphasia by noninvasive brain stimulation. Front Hum Neurosci 2013; 7: 888.

16. Elsner B, Kugler J, Pohl M, Mehrholz J. Transcranial direct current stimulation (tDCS) for improving aphasia in patients with aphasia after stroke. Cochrane Database Syst Rev 2015; 5: CD009760.

17. Miniussi C, Cappa SF, Cohen LG, Floel A, Fregni F, Nitsche MA, et al. Efficacy of repetitive transcranial magnetic stimulation/transcranial direct current stimulation in cognitive neurorehabilitation. Brain Stimul 2008; 1: 326-36.
18. Alharbi MF, Armijo-Olivo S, Kim ES. Transcranial direct current stimulation (tDCS) to improve naming ability in post-stroke aphasia: a critical review. Behav Brain Res 2017; 332: 7-15.

19. Thair H, Holloway AL, Newport R, Smith AD. Transcranial direct current stimulation (tDCS): a beginner's guide for design and implementation. Front Neurosci 2017; 11: 641.

20. Turski CA, Kessler-Jones A, Chow C, Hermann B, Hsu D, Jones J, et al. Extended multiple-field high-definition transcranial direct current stimulation (HD-tDCS) is well tolerated and safe in healthy adults. Restor Neurol Neurosci 2017; 35: 631-42.

21. De Aguiar V, Bastiaanse R, Capasso R, Gandolfi M, Smania N, Rossi G, et al. Can tDCS enhance item-specific effects and generalization after linguistically motivated aphasia therapy for verbs? Front Behav Neurosci 2015; 9: 190.

22. Palm U, Keeser D, Schiller C, Fintescu Z, Nitsche M, Reisinger E, et al. Skin lesions after treatment with transcranial direct current stimulation (tDCS). Brain Stimul 2008; 1: 386-7.

23. Bikson M, Grossman P, Thomas C, Zannou AL, Jiang J, Adnan T, et al. Safety of transcranial direct current stimulation: evidence based update 2016. Brain Stimul 2016; 9: 641-61.

24. Brunoni AR, Schestatsky P, Lotufo PA, Benseñor IM, Fregni F. Comparison of blinding effectiveness between sham tDCS and placebo sertraline in a 6-week major depression randomized clinical trial. Clin Neurophysiol 2014; 125: 298-305.

25. Floel A. tDCS-enhanced motor and cognitive function in neurological diseases. Neuroimage 2014; 85: 934-47.

26. Antal A, Terney D, Kuhnl S, Paulus W. Anodal transcranial direct current stimulation of the motor cortex ameliorates chronic pain and reduces short intracortical inhibition. J Pain Symptom Manage 2010; 39: 890-903.

27. Cattaneo Z, Pisoni A, Papagno C. Transcranial direct current stimulation over Broca's region improves phonemic and semantic fluency in healthy individuals. Neuroscience 2011; 183: 64-70

28. Fertonani A, Rosini S, Cotelli M, Rossini PM, Miniussi C. Naming facilitation induced by transcranial direct current stimulation. Behav Brain Res 2010; 208: 311-8.

29. Sparing R, Dafotakis M, Meister IG, Thirugnanasambandam N, Fink GR. Enhancing language performance with non-invasive brain stimulation $-\mathrm{a}$ transcranial direct current stimulation study in healthy humans. Neuropsychologia 2008; 46: 261-8.

30. Elsner B, Kugler J, Pohl M, Mehrholz J. Transcranial direct current stimulation (tDCS) for improving aphasia in patients after stroke. Cochrane Database Syst Rev 2013; 6: CD009760.

31. Otal B, Olma MC, Floel A, Wellwood I. Inhibitory non-invasive brain stimulation to homologous language regions as an adjunct to speech and language therapy in post-stroke aphasia: a meta-analysis. Front Hum Neurosci 2015; 9: 236.

32. Lefaucheur JP, Antal A, Ayache SS, Benninger DH, Brunelin J, Cogiamanian F, et al. Evidence-based guidelines on the therapeutic use of transcranial direct current stimulation (tDCS). Clin Neurophysiol 2017; 128: 56-92.

33. Biou E, Cassoudesalle H, Cogné M, Sibon I, De Gabory I, Dehail P, et al. Transcranial direct current stimulation in post-stroke aphasia rehabilitation: a systematic review. Ann Phys Rehabil 2019; 62: 104-21.

34. Elsner B, Kugler J, Pohl M, Mehrholz J. Transcranial direct current stimulation for improving aphasia after stroke -what's the current evidence? Stroke 2019; 50: E248-9.

35. Elsner B, Kugler J, Pohl M, Mehrholz J. Transcranial direct current stimulation (tDCS) for improving function and activities of daily living in patients after stroke. Cochrane Database Syst Rev 2013; 11: CD009645.

36. Rosso C, Arbizu C, Dhennain C, Lamy J, Samson Y. Repetitive sessions of tDCS to improve naming in post-stroke aphasia: insights from an individual patient data (IPD) metaanalysis. Restor Neurol Neurosci 2018; 36: 107-16.

37. Urrutia G, Bonfill X. PRISMA declaration: a proposal to improve the publication of systematic reviews and metaanalyses. Med Clin (Barc) 2010; 135: 507-11.

38. Anglade C, Thiel A, Ansaldo AI. The complementary role 
of the cerebral hemispheres in recovery from aphasia after stroke: a critical review of literature. Brain Inj 2014; 28: 138-45.

39. Vines BW, Norton AC, Schlaug G. Non-invasive brain stimulation enhances the effects of melodic intonation therapy. Front Psychol 2011; 2: 230.

40. Winhuisen L, Thiel A, Schumacher B, Kessler J, Rudolf J, Haupt WF, et al. The right inferior frontal gyrus and poststroke aphasia: a follow-up investigation. Stroke 2007; 38: 1286-92.

41. Saur D, Lange R, Baumgaertner A, Schraknepper V, Willmes K, Rijntjes M, et al. Dynamics of language reorganization after stroke. Brain 2006; 129: 1371-84.

42. Torres J, Drebing D, Hamilton R. TMS and tDCS in post-stroke aphasia: integrating novel treatment approaches with mechanisms of plasticity. Rest Neurol Neurosci 2013; 31: 501-15.

43. Norise C, Sacchetti D, Hamilton R. Transcranial direct current stimulation in post-stroke chronic aphasia: the impact of baseline severity and task specificity in a pilot sample. Front Hum Neurosci 2017; 11: 260.

44. Marsh E, Hillis A. Recovery from aphasia following brain injury: the role of reorganization. Prog Brain Res 2006; 157: 143-56.

45. Fregni F, Thome-Souza S, Nitsche MA, Freedman SD, Valente KD, Pascual-Leone A. A controlled clinical trial of cathodal DC polarization in patients with refractory epilepsy. Epilepsia 2006; 47: 335-42.

46. Burn J, Dennis M, Bamford J, Sandercock P, Wade D, Warlow C. Epileptic seizures after a first stroke: the Oxfordshire community stroke project. BMJ 1997; 315: 1582-7.

47. Polanowska KE, Lesniak MM, Seniow JB, Czepiel W, Czlonkowska A. Anodal transcranial direct current stimulation in early rehabilitation of patients with post-stroke non-fluent aphasia: a randomized, double-blind, sham-controlled pilot study. Restor Neurol Neurosci 2013; 31: 761-71.

48. Vines BW, Cerruti C, Schlaug G. Dual-hemisphere tDCS facilitates greater improvements for healthy subjects' nondominant hand compared to uni-hemisphere stimulation. BMC Neurosci 2008; 9: 103.

49. Baker JM, Rorden C, Fridriksson J. Using transcranial direct current stimulation to treat stroke patients with aphasia. Stroke 2010; 41: 1229-36.

50. Fiori V, Coccia M, Marinelli CV, Vecchi V, Bonifazi S, Ceravolo MG, et al. Transcranial direct current stimulation improves word retrieval in healthy and nonfluent aphasic subjects. J Cogn Neurosci 2011; 23: 2309-23.

51. Floël A, Meinzer M, Kirstein R, Nijhof S, Deppe M, Knecht S, et al. Short-term anomia training and electrical brain stimulation. Stroke 2011; 42: 2065-7.

52. Fridriksson J, Richardson JD, Baker JM, Rorden C. Transcranial direct current stimulation improves naming reaction time in fluent aphasia: a double-blind, sham-controlled study. Stroke 2011; 42: 819-21.

53. Kang EK, Kim YK, Sohn HM, Cohen LG, Paik NJ. Improved picture naming in aphasia patients treated with cathodal tDCS to inhibit the right Broca's homologue area. Restor Neurol Neurosci 2011; 29: 141-52.

54. Marangolo P, Marinelli CV, Bonifazi S, Fiori V, Ceravolo MG, Provinciali L, et al. Electrical stimulation over the left inferior frontal gyrus (IFG) determines long-term effects in the recovery of speech apraxia in three chronic aphasics. Behav Brain Res 2011; 225: 498-504.

55. You DS, Kim DY, Chun MH, Jung SE, Park SJ. Cathodal transcranial direct current stimulation of the right Wernicke's area improves comprehension in subacute stroke patients. Brain Lang 2011; 119: 1-5.

56. Marangolo P, Fiori V, Di Paola M, Cipollari S, Razzano C, Oliveri M, et al. Differential involvement of the left frontal and temporal regions in verb naming: a tDCS treatment study. Restor Neurol Neurosci 2013; 31: 63-72.

57. Marangolo P, Fiori V, Cipollari S, Campana S, Razzano C, Di Paola M, et al. Bihemispheric stimulation over left and right inferior frontal region enhances recovery from apraxia of speech in chronic aphasia. Eur J Neurosci 2013; 38: 3370-7.

58. Marangolo P, Fiori V, Calpagnano MA, Campana S, Razzano C,
Caltagirone C, et al. tDCS over the left inferior frontal cortex improves speech production in aphasia. Front Hum Neurosci 2013; 7: 539.

59. Fiori V, Cipollari S, Di Paola M, Razzano C, Caltagirone C, Marangolo P. tDCS stimulation segregates words in the brain: evidence from aphasia. Front Hum Neurosci 2013; 7: 269.

60. Lee SY, Cheon H, Yoon KJ, Chang WH, Kim Y. Effects of dual transcranial direct current stimulation for aphasia in chronic stroke patients. Ann Rehabil Med 2013; 37: 603-10.

61. Polanowska KE, Lesniak M, Seniow JB, Czlonkowska A. No effects of anodal transcranial direct stimulation on language abilities in early rehabilitation of post-stroke aphasic patients. Neurol Neurochir Pol 2013; 47: 414-22.

62. Santos MD, Gagliardi RJ, Mac-Kay AP, Boggio PS, Lianza R, Fregni F. Transcranial direct-current stimulation induced in stroke patients with aphasia: a prospective experimental cohort study. Sao Paulo Med J 2013; 131: 422-6.

63. Volpato C, Cavinato M, Piccione F, Garzon M, Meneghello F, Birbaumer N. Transcranial direct current stimulation (tDCS) of Broca's area in chronic aphasia: a controlled outcome study. Behav Brain Res 2013; 247: 211-6.

64. Marangolo P, Caltagirone C. Options to enhance recovery from aphasia by means of non-invasive brain stimulation and action observation therapy. Expert Rev Neurother 2014; 14: 75-91.

65. Rosso C, Perlbarg V, Valabregue R, Arbizu C, Ferrieux S, Alshawan B, et al. Broca's area damage is necessary but not sufficient to induce after-effects of cathodal tDCS on the unaffected hemisphere in post-stroke aphasia. Brain Stimul 2014; 7: 627-35.

66. Vestito L, Rosellini S, Mantero M, Bandini F. Long-term effects of transcranial direct-current stimulation in chronic post-stroke aphasia: a pilot study. Front Hum Neurosci 2014; 8: 785.

67. Campana S, Caltagirone C, Marangolo P. Combining voxelbased lesion-symptom mapping (VLSM) with A-tDCS language treatment: predicting outcome of recovery in nonfluent chronic aphasia. Brain Stimul 2015; 8: 769-76.

68. Cipollari S, Veniero D, Razzano C, Caltagirone C, Koch G, Marangolo P. Combining TMS-EEG with transcranial direct current stimulation language treatment in aphasia. Expert Rev Neurother 2015; 15: 833-45.

69. Shah-Basak PP, Norise C, García G, Torres J, Faseyitan O, Hamilton RH. Individualized treatment with transcranial direct current stimulation in patients with chronic non-fluent aphasia due to stroke. Front Hum Neurosci 2015; 9: 201.

70. Wu D, Wang J, Yuan Y. Effects of transcranial direct current stimulation on naming and cortical excitability in stroke patients with aphasia. Neurosci Lett 2015; 589: 115-20.

71. Lifshitz Ben Basat A, Gvion A, Vatine J, Mashal N. Transcranial direct current stimulation to improve naming abilities of persons with chronic aphasia: a preliminary study using individualized based protocol. J Neurolinguistics 2016; 38: $1-13$.

72. Marangolo P, Fiori V, Sabatini U, De Pasquale G, Razzano C, Caltagirone $\mathrm{C}$, et al. Bilateral transcranial direct current stimulation language treatment enhances functional connectivity in the left hemisphere: preliminary data from aphasia. J Cogn Neurosci 2016; 28: 724-38.

73. Meinzer M, Darkow R, Lindenberg R, Flöel A. Electrical stimulation of the motor cortex enhances treatment outcome in post-stroke aphasia. Brain 2016; 139: 1152-63.

74. Darkow R, Martin A, Würtz A, Flöel A, Meinzer M. Transcranial direct current stimulation effects on neural processing in post-stroke aphasia. Hum Brain Mapp 2017; 38: 1518-31.

75. Dos Santos MD, Cavenaghi VB, Mac-Kay APMG, Serafim V, Venturi A, Truong DQ, et al. Non-invasive brain stimulation and computational models in post-stroke aphasic patients: single session of transcranial magnetic stimulation and transcranial direct current stimulation. A randomized clinical trial. Sao Paulo Med J 2017; 135: 475-80.

76. Fridriksson J, Rorden C, Elm J, Sen S, George M, Bonilha L. Transcranial direct current stimulation vs sham stimulation to 
treat aphasia after stroke. A randomized clinical trial. JAMA Neurol 2018; 75: 1470-6.

77. Branscheidt M, Hoppe J, Zwitserlood P, Liuzzi G. tDCS over the motor cortex improves lexical retrieval of action words in poststroke aphasia. J Neurophysiol 2018; 119: 621-30.

78. Marangolo P, Fiori V, Caltagirone C, Pisano F, Priori A. Transcranial cerebellar direct current stimulation enhances verb generation but not verb naming in poststroke aphasia. J Cogn Neurosci 2018; 30: 188-99.

79. Pestalozzi MI, Di Pietro M, Martins Gaytanidis C, Spierer L, Schnider A, Chouiter L, et al. Effects of prefrontal transcranial direct current stimulation on lexical access in chronic poststroke aphasia. Neurorehabil Neural Repair 2018; 32: 913-23.

80. Geschwind N. Current concepts: aphasia. N Engl J Med 1971; 284: 654 .

81. Marangolo P, Cipollari S, Fiori V, Razzano C, Caltagirone C. Walking but not barking improves verb recovery: implications for action observation treatment in aphasia rehabilitation. PLoS One 2012; 7: e38610.

82. Friederici AD. The brain basis of language processing: from structure to function. Physiol Rev 2011; 91: 1357-92.

83. Bastiaanse R, Jonkers R, Quak C, Varela Put M. Werkwoord- productie op Woorden Zinsniveau [verb production at the word and sentence level]. Lisse: Swets \& Zeitlinger; 1997.

84. Nitsche M, Paulus W. Sustained excitability elevations induced by transcranial DC motor cortex stimulation in humans. Neurology 2001; 57: 1899-901.

85. Holland R, Crinion J. Can tDCS enhance treatment of aphasia after stroke? Aphasiology 2012; 26: 1169-91.

86. Pulvermüller F, Neininger B, Elbert T, Mohr B, Rockstroh B, Koebbel $\mathrm{P}$, et al. Constraint-induced therapy of chronic aphasia after stroke. Stroke 2001; 32: 1621-6.

87. Rosell-Clari V, Hernández-Sacristán C. Rehabilitación de la anomia desde una perspectiva pragmática-funcional. Revista de Investigación en Logopedia 2017; 7: 47-70.

88. Cuetos-Vega F. Rehabilitación de la anomia mediante un programa informático. Revista Española de Neuropsicología 2003; 5: 199-211.

89. Cuetos F, González-Nostia M, Sánchez N, Griffith H, Cabezas C, García P, et al. Tipos de trastornos anómicos en las afasias. Revista de Logopedia, Foniatría y Audiología 2010; 30: 94-9.

90. Montenegro IR, Álvarez-Montesinos JA, Estudillo AJ, GarcíaOrza J. Estimulación eléctrica por corriente continua en el tratamiento de la afasia. Rev Neurol 2017; 553: 62.

\section{Transcranial direct current stimulation and speech therapy intervention in people with aphasia: a systematic review of the literature}

Introduction. The recovery of aphasia may require adjuvant therapies to speech therapy rehabilitation. Transcranial direct current stimulation (tDCS) is a non-invasive neuromodulatory technique that can be used to improve cortical brain activity.

Aim. To offer an overview of the tDCS in people with aphasia from a speech therapy point of view.

Patients and methods. Thirty-five studies have been analyzed that performed online interventions (the application of tDCS in conjunction with any type of aphasia therapy) and offline interventions (performing speech therapy training before or after tDCS stimulation). The studies were published during the last ten years (January 2009-2019). PubMed, Scopus and Embase databases were reviewed in the analysis.

Results. Most studies include heterogeneous samples without taking into account the symptoms of each type of aphasia and without individualizing the treatments used. Anomie is the most commonly treated language deficit.

Conclusions. It is better to perform tDCS in conjunction with online speech therapy. The tDCS is a technique that can help improve the language deficits of people with aphasia. Speech therapy interventions based on neuroscientific knowledge that have an impact on the daily life of people with aphasia should be performed.

Key words. Aphasia. Language. Speech therapy. Stroke. tDCS. Transcranial direct current stimulation. 